\title{
openheart Patient perception of anticoagulant treatment for stroke prevention (RE-SONANCE study)
}

\author{
Dragos Vinereanu, ${ }^{1}$ Dmitry Napalkov, ${ }^{2}$ Jutta Bergler-Klein, ${ }^{3}$ Bela Benczur, ${ }^{4}$ \\ Martin Ciernik, ${ }^{5}$ Nina Gotcheva, ${ }^{6}$ Alexey Medvedchikov (D) , ${ }^{5}$ Pentti Põder, ${ }^{7}$ \\ Dragan Simic, ${ }^{8}$ Andris Skride, ${ }^{9}$ Wenbo Tang, ${ }^{10}$ Maria Trusz-Gluza, ${ }^{11}$ Jiri Vesely, ${ }^{12}$ \\ Tatiana Vishnepolsky, ${ }^{13}$ Mirej Vrabec ${ }^{14}$
}

\begin{abstract}
- Additional material is published online only. To view please visit the journal online (http://dx.doi.org/10.1136/ openhrt-2019-001202).
\end{abstract}

To cite: Vinereanu D, Napalkov D, Bergler-Klein J, et al. Patient perception of anticoagulant treatment for stroke prevention (RE-SONANCE study). Open Heart 2020;7:e001202. doi:10.1136/ openhrt-2019-001202

Received 30 0ctober 2019 Revised 20 December 2019 Accepted 10 February 2020
Check for updates

\section{(c) Author(s) (or their} employer(s)) 2020. Re-use permitted under CC BY. Published by BMJ.

For numbered affiliations see end of article.

\section{Correspondence to}

Professor Dragos Vinereanu; vinereanu@gmail.com

\section{ABSTRACT}

Objective We evaluated atrial fibrillation (AF) patients' perceptions of anticoagulation treatment with dabigatran or a vitamin $\mathrm{K}$ antagonist (VKA) for stroke prevention, according to accepted indications.

Methods The RE-SONANCE observational, prospective, multicentre, international study used the validated Perception on Anticoagulant Treatment Questionnaire (PACT-Q) to assess patients with AF already taking a VKA who were switched to dabigatran (cohort $A$ ), and newly diagnosed patients initiated on either dabigatran or a VKA (cohort B). Visit 1 (V1) was at baseline, and visit 2 (V2) and visit 3 (V3) were at 30-45 and 150-210 days after baseline, respectively. Primary outcomes were treatment satisfaction and convenience in cohort A at V2 and V3 versus baseline, and in cohort $B$ for dabigatran and a VKA at V2 and V3.

Results The main analysis set comprised 4100 patients in cohort $A$ and 5365 in cohort $B$ (dabigatran: 3179; VKA: 2186). In cohort A, PACT-Q2 improved significantly $(p<0.001$ for all) for treatment convenience (mean change V1 vs V2=20.72; $S D=21.50 ;$ V1 vs V3=24.54; $S D=22.85$ ) and treatment satisfaction (mean change V1 vs V2 $=17.60$; $\mathrm{SD}=18.76$; V1 vs V3=21.04; $S D=20.24$ ). In cohort $B$, mean PACT-Q2 scores at V2 and V3 were significantly higher $(p<0.001$ for all) for dabigatran versus a VKA for treatment convenience (V2=18.38; $\mathrm{SE}=0.51$; $\mathrm{V} 3=23.34$; $\mathrm{SE}=0.51$ ) and satisfaction $(\mathrm{V} 2=15.88$; $\mathrm{SE}=0.39 ; \mathrm{V} 3=19.01$; $\mathrm{SE}=0.41$ ).

Conclusions Switching to dabigatran from long-term VKA therapy or newly initiated dabigatran is associated with improved patient treatment convenience and satisfaction compared with VKA therapy.

\section{INTRODUCTION}

Prior to the availability of novel oral anticoagulants (NOACs), vitamin $\mathrm{K}$ antagonists (VKAs) such as warfarin were the mainstay of anticoagulation therapy for prevention of stroke in non-valvular atrial fibrillation (NVAF). However, there is a real-world underuse of oral anticoagulants (OACs) in patients with atrial fibrillation (AF) who should be receiving treatment, resulting in

\section{Key questions}

What is already known about this subject?

- Including patients in their anticoagulant treatment decision-making is important, and using educational intervention programmes targeting both patients and physicians can improve the use of oral anticoagulation in patients with atrial fibrillation (AF) who are at risk of stroke. Evaluating patients' perspectives of anticoagulation treatment satisfaction is also important, as this may impact adherence and therefore outcomes. Nevertheless, data on patients perception of long-term anticoagulation therapy in non-valvular AF (NVAF) are limited.

What does this study add?

- The RE-SONANCE observational prospective study evaluated the perceptions of anticoagulation treatment and treatment convenience of patients with NVAF treated with dabigatran or a vitamin $\mathrm{K}$ antagonist (VKA) for stroke prevention, according to accepted indications.

How might this impact on clinical practice?

- Switching to dabigatran from long-term VKA therapy or newly initiated dabigatran is associated with improved patient treatment convenience and satisfaction compared with VKA therapy. This may be important in the prevention of stroke and systemic embolism in patients with AF patients at moderateto-high risk.

a great number of preventable ischaemic strokes in these undertreated patients. ${ }^{1}$ Several factors contributed to suboptimal adherence with VKAs, including the narrow therapeutic window of VKAs, numerous food and drug interactions, a variable doseresponse relationship and the requirement for frequent anticoagulation monitoring and dose adjustment with VKAs. The NOACs circumvent many of these problems and are currently recommended by European guidelines as the preferred anticoagulation treatment in patients with $\mathrm{AF}$ at risk of stroke. ${ }^{2-4}$ 
Including patients in their treatment decision-making is important, ${ }^{3}$ and using educational intervention programmes targeting both patients and physicians can improve the use of OACs in patients with $\mathrm{AF}$ at risk of stroke. ${ }^{5-9}$ Evaluating patients' perspectives of anticoagulation treatment satisfaction is also important, as this may impact adherence and therefore outcomes. ${ }^{10}$ However, data on patients' perception of long-term anticoagulation therapy in NVAF are limited. ${ }^{511-13}$

Therefore, we have evaluated perceptions of anticoagulation treatment and treatment convenience of patients with NVAF treated with dabigatran or a VKA for stroke prevention, according to accepted indications. The RE-SONANCE observational prospective study (NCT02684981), conducted in 11 European countries and Israel, used the validated Perception on Anticoagulant Treatment Questionnaire (PACT-Q) $)^{10}$ to assess two different patient cohorts: patients with NVAF taking a VKA who were switched to dabigatran and newly diagnosed patients with NVAF initiated on either dabigatran or a VKA.

\section{METHODS}

\section{Study population and trial design}

The study was conducted in Austria, Bulgaria, Czech Republic, Estonia, Hungary, Israel, Latvia, Poland, Romania, Russian Federation, Serbia and Slovenia. Eligible patients were $\geq 18$ years old with NVAF and an indication for anticoagulation therapy for stroke prevention, not participating in any other clinical trial (for a drug or device) or registry. Cohort A included patients with NVAF switched from a VKA to dabigatran $(\geq 3$ months continuous VKA treatment for stroke prevention prior to baseline), while cohort B included newly diagnosed patients with NVAF initiated on either dabigatran or a VKA (with no use of any OAC within 1 year prior to enrolment).

\section{Assessments}

Collection of patient characteristics and treatment data was managed during routine clinic visits at three recommended time points. Visit 1 (V1) was at baseline, when patients were either switched from a VKA to dabigatran or started on dabigatran or VKA treatment. Visit 2 (V2) was 30-45 days after baseline. Visit 3 (V3) was 150-210 days after baseline. While these proposed time periods were provided as guidance to the treating physician, the timing of each visit was based on real-life practice in the respective countries. Therefore, visits were also performed by sites outside these windows, so the time points were revised and extended to: V2, 7-124 days after baseline and V3, 125-365 days after baseline. PACT-Q was used as a self-administered questionnaire. ${ }^{10}{ }^{14}$ Patient expectations regarding their anticoagulant treatment were assessed using PACT-Q1. PACT-Q2 assessed perceptions regarding convenience, anticoagulant treatment satisfaction, burden of disease and treatment. Cohort A completed PACT-Q2 at baseline, V2 and V3, and cohort B completed PACT-Q1 at baseline and PACT-Q2 at V2 and V3.

\section{Outcomes}

Primary outcomes

The primary outcome for cohort A was patient satisfaction with anticoagulant treatment and treatment convenience (PACT-Q2 domains) at V2 and V3 versus baseline. For cohort B, it was patient satisfaction with anticoagulant treatment and treatment convenience (PACT-Q2 domains) between treatment groups (dabigatran vs VKA) at V2 and V3.

\section{Secondary outcomes}

For cohort A, this was to evaluate the mean changes over time in patients' satisfaction and treatment convenience (PACT-Q2 domain) between V3 and V2; for cohort B, it was anticoagulation treatment expectations (PACT-Q1 items) at baseline.

\section{Safety outcomes}

Safety was evaluated separately for patients in cohorts A and $\mathrm{B}$ with follow-up for potential adverse drug reactions (ADRs) and fatal adverse events.

\section{Statistical analyses}

Sample size and analysis sets

The planned sample size was 9000 patients. For cohort A (assuming a two-sided alpha of 0.05 and that $20 \%$ of patients would be lost to follow-up), a total sample size of 3000 patients would provide $>80 \%$ power to detect a standardised mean difference of 0.06 in PACT-Q2 scores between two assessments. For cohort B (assuming a two-sided alpha of 0.05 , a 1:1 ratio of dabigatran and VKA patients and a $30 \%$ loss to follow-up and matching), a total sample size of 6000 patients would provide $>80 \%$ power to detect a standardised mean difference of 0.065 in PACT-Q2 scores between dabigatran and a VKA at each assessment.

Enrolled patients were those who met all eligibility criteria. The main analysis set (MAS) comprised all eligible patients with known treatment; the safety analysis set comprised all enrolled patients with follow-up data. The propensity score matched set (PSMS) compared PACT-Q2 treatment differences between dabigatran and a VKA in cohort B; all patients matched with a 1:n (n, range 1-3) ratio (VKA:dabigatran) based on propensity scores calculated using a logistic regression model. Patients who permanently discontinued initial anticoagulation treatment at the time of an assessment were excluded from all analyses where data from that assessment were included.

\section{Assessment of PACT-Q2 scores}

For cohort A, mean differences in PACT-Q2 scores between visits were assessed using paired t-tests. In cohort $\mathrm{B}$, mean differences in PACT-Q2 scores between treatment groups were assessed using the PSMS and the random intercept model. Paired t-tests were used for sensitivity analysis. The 
baseline variables used in the PSMS included: sex (men/ women); age $(<65 / \geq 65$ to $<75 / \geq 75$ years $)$; reimbursement status (reimbursed/partially reimbursed/private pay/other); physician specialty (cardiologist/internist/ neurologist/general practitioner/other); HAS-BLED score (low $(<3) /$ high $(\geq 3)$ risk); $\mathrm{CHA}_{2} \mathrm{DS}_{2}$-VASc score (low or intermediate $(<2) /$ high $(\geq 2)$ risk); number of concomitant medications $(0 / 1-3 / \geq 4)$; type of concomitant medication (prescription/no prescription of (i) antiarrhythmics, (ii) antiplatelets or (iii) non-steroidal antiinflammatory drugs); number of concomitant therapies
$(0 / \geq 1)$; presence of comorbidities (presence/absence of (i) malignancy or (ii) gastro-oesophageal reflux disease or gastroduodenal ulcer disease). The planned primary analysis was originally based on a 1:1 patient ratio. However, unequal enrolment (reflecting real-world treatment patterns and country recruitment) led to an adjustment of this analysis. Therefore, due to the variable size of the matched sets, the primary analysis of PACT-Q2 scores was based on the random intercept model, where a variance in the unequal groups (1:n matching) is compared and one group contains 'repeated' observations.

Table 1 Baseline demographics, comorbidities and concomitant medications (main analysis set)

\begin{tabular}{|c|c|c|c|c|}
\hline & \multicolumn{2}{|c|}{ Cohort A (switched from VKA to dabigatran) } & \multicolumn{2}{|c|}{$\begin{array}{l}\text { Cohort B (newly initiated on dabigatran or } \\
\text { a VKA) }\end{array}$} \\
\hline & Total $(n=4100)$ & Dabigatran $(n=3179)$ & VKA $(n=2186)$ & Total $(n=5365)$ \\
\hline Mean age, years (SD) & $70.5(9.6)$ & $68.6(10.1)$ & $68.5(9.5)$ & $68.6(9.9)$ \\
\hline Range, years & $18-100$ & 22-95 & 18-95 & 18-95 \\
\hline \multicolumn{5}{|l|}{ Age, n (\%) } \\
\hline$<65$ years & $1029(25.1)$ & $1042(32.8)$ & $723(33.1)$ & $1765(32.9)$ \\
\hline 65 to $<75$ years & $1552(37.9)$ & $1154(36.3)$ & $803(36.7)$ & $1957(36.5)$ \\
\hline$\geq 75$ years & $1519(37.0)$ & $983(30.9)$ & $660(30.2)$ & $1643(30.6)$ \\
\hline \multicolumn{5}{|l|}{ Sex, n (\%) } \\
\hline Female & $1998(48.7)$ & $1602(50.4)$ & $1080(49.4)$ & $2682(50.0)$ \\
\hline Male & $2102(51.3)$ & $1577(49.6)$ & $1106(50.6)$ & $2683(50.0)$ \\
\hline Mean (SD) weight, kg & $84.1(16.2)$ & $82.8(15.5)$ & $82.5(15.0)$ & $82.6(15.3)$ \\
\hline Comorbidities, n (\%) & $3541(86.4)$ & 2651 (83.4) & $1986(90.9)$ & 4637 (86.4) \\
\hline Blood and immune system & $62(1.5)$ & $40(1.3)$ & $29(1.3)$ & $69(1.3)$ \\
\hline Gl/metabolic & $1618(39.5)$ & $1044(32.8)$ & $774(35.4)$ & $1818(33.9)$ \\
\hline Heart and blood vessels & $3390(82.7)$ & $2548(80.2)$ & $1904(87.1)$ & $4452(83.0)$ \\
\hline Previous Ml & $95(2.3)$ & $77(2.4)$ & $78(3.6)$ & $155(2.9)$ \\
\hline CVA/previous TIA & $247(6.0)$ & $168(5.3)$ & $118(5.4)$ & $286(5.3)$ \\
\hline Previous CHD & $1048(25.6)$ & $749(23.6)$ & $625(28.6)$ & $1374(25.6)$ \\
\hline Hypertension (arterial) & 2921 (71.2) & $2228(70.1)$ & $1689(77.3)$ & $3917(73.0)$ \\
\hline Lung & $285(7.0)$ & $166(5.2)$ & $131(6.0)$ & $297(5.5)$ \\
\hline Malignancy & $48(1.2)$ & $28(0.9)$ & $7(0.3)$ & $35(0.7)$ \\
\hline Urogenital system & $308(7.5)$ & $217(6.8)$ & $175(8.0)$ & $392(7.3)$ \\
\hline Other* & $632(15.4)$ & $416(13.1)$ & $270(12.4)$ & $686(12.8)$ \\
\hline Concomitant medications, $\mathrm{n}(\%)$ & $3542(86.4)$ & $652(83.4)$ & $1993(91.2)$ & $4645(86.6)$ \\
\hline Antacids & $393(9.6)$ & $248(7.8)$ & $167(7.6)$ & $415(7.7)$ \\
\hline Antidiabetes drugs & $687(16.8)$ & 392 (12.3) & $344(15.7)$ & $736(13.7)$ \\
\hline Antihypertensive drugs & $3229(78.8)$ & $2428(76.4)$ & $1839(84.1)$ & $4267(79.5)$ \\
\hline Antiarrhythmic drugs & $1479(36.1)$ & $1052(33.1)$ & $805(36.8)$ & $1857(34.6)$ \\
\hline Antiplatelet drugs & $274(6.7)$ & $307(9.7)$ & $275(12.6)$ & $582(10.8)$ \\
\hline Lipid-lowering drugs & $1670(40.7)$ & $1227(38.6)$ & $920(42.1)$ & $2147(40.0)$ \\
\hline NSAIDs & $45(1.1)$ & $34(1.1)$ & $15(0.7)$ & $49(0.9)$ \\
\hline Other & 898 (21.9) & 487 (15.3) & 355 (16.2) & 842 (15.7) \\
\hline
\end{tabular}

*Includes depression, psoriasis, thyropathy, vertebral algic syndrome and osteoporosis.

CHD, coronary heart disease; CVA, cerebrovascular accident; Gl, gastrointestinal; MI, myocardial infarction; NSAIDs, non-steroidal antiinflammatory drugs; TIA, transient ischaemic attack; VKA, vitamin $\mathrm{K}$ antagonist. 


\section{Assessment of PACT-Q1 scores}

PACT-Q1 scores in cohort B at baseline were summarised descriptively for all patients and between treatment groups.

\section{RESULTS}

\section{Patient disposition}

In total, 9472 patients with NVAF were enrolled from 698 sites in 11 European countries and Israel. The flow of patients through the study is summarised in online appendix figure 1. A total of 577 patients $(240(5.9 \%)$ in cohort A; 337 in cohort B (210 (6.6\%) receiving dabigatran and 127 (5.8\%) receiving a VKA)) permanently discontinued treatment during the study or had unknown treatment status at the end of observation. In cohort B, of those patients discontinuing VKA therapy, 42 (1.9\%) were switched to dabigatran. All data collected prior to premature discontinuation were included in the analyses.

\section{Patient characteristics and anticoagulation treatment}

Tables 1-3 summarise the demographic and baseline characteristics, healthcare system characteristics and physician-rated risk scores in the MAS. Most patients had a high risk of stroke/bleeding complications, had comorbidities and were receiving concomitant medications. Creatinine clearance (calculated at each visit using the Cockcroft-Gault equation) was stable in both cohorts (data not shown). There was a high risk of stroke or systemic embolism $\left(\mathrm{CHA}_{2} \mathrm{DS}_{2}-\mathrm{VASc}\right.$ score $\left.\geq 2\right)$ and bleeding complications (HAS-BLED score $\geq 3$ ), reported in $88 \%$ and $59 \%$ of patients in cohort A, $88 \%$ and $29 \%$ of cohort $\mathrm{B}$ dabigatran-treated patients and $91 \%$ and $31 \%$ of cohort B VKA-treated patients, respectively.

\begin{tabular}{|c|c|c|c|c|}
\hline & \multicolumn{2}{|c|}{$\begin{array}{l}\text { Cohort A (switched from } \\
\text { VKA to dabigatran) }\end{array}$} & \multicolumn{2}{|c|}{$\begin{array}{l}\text { Cohort B (newly } \\
\text { initiated on dabigatran } \\
\text { or a VKA) }\end{array}$} \\
\hline & $\begin{array}{l}\text { Total } \\
(n=4100)\end{array}$ & $\begin{array}{l}\text { Dabigatran } \\
(\mathrm{n}=3179)\end{array}$ & $\begin{array}{l}\text { VKA } \\
(n=2186)\end{array}$ & $\begin{array}{l}\text { Total } \\
(n=5365)\end{array}$ \\
\hline \multicolumn{5}{|c|}{ Specialty of the treating physician, $n(\%)$} \\
\hline Cardiologist & $3230(78.8)$ & $2682(84.4)$ & 1935 (88.5) & $4617(86.1)$ \\
\hline $\begin{array}{l}\text { General } \\
\text { practitioner }\end{array}$ & $96(2.3)$ & $82(2.6)$ & $27(1.2)$ & $109(2.0)$ \\
\hline Internist & $607(14.8)$ & $202(6.4)$ & $90(4.1)$ & $292(5.4)$ \\
\hline Neurologist & $153(3.7)$ & $198(6.2)$ & $126(5.8)$ & $324(6.0)$ \\
\hline Other & $14(0.3)$ & $15(0.5)$ & $8(0.4)$ & $23(0.4)$ \\
\hline \multicolumn{5}{|c|}{ Healthcare reimbursement status, $n(\%)$} \\
\hline $\begin{array}{l}\text { Partially } \\
\text { reimbursed }\end{array}$ & $490(12.0)$ & $26(10.3)$ & $342(15.6)$ & $668(12.5)$ \\
\hline Private pay & $1430(34.9)$ & $1614(50.8)$ & $1018(46.6)$ & $2632(49.1)$ \\
\hline Reimbursed & $2153(52.5)$ & $1197(37.7)$ & $808(37.0)$ & 2005 (37.4) \\
\hline Other & $27(0.7)$ & $42(1.3)$ & $18(0.8)$ & $60(1.1)$ \\
\hline
\end{tabular}

VKA, vitamin $\mathrm{K}$ antagonist.
In both cohorts, most patients had comorbidities and were receiving concomitant medications: $86 \%$ of patients in cohorts $\mathrm{A}$ and $\mathrm{B}$, and $83 \%$ and $91 \%$ of dabigatran and VKA-treated patients, respectively. The most frequently prescribed concomitant medication was antihypertensives, most of which were angiotensin-converting enzyme inhibitors (used by 1565 (38.2\%) (cohort A) and 2119 $(39.5 \%)$ (cohort B) patients). Most lipid-lowering agents were statins (used by 1620 (39.5\%) (cohort A) and 2104 (39.2\%) (cohort B) patients). Antiarrhythmic agents were mostly class III potassium channel blockers (used by $474(11.6 \%)$ (cohort A) and $688(12.8 \%)$ (cohort B) patients), although beta-adrenergic receptor blockers were used as antiarrhythmics in $736(18.0 \%)$ patients in cohort $\mathrm{A}$ and $954(17.8 \%)$ in cohort $\mathrm{B}$. The most frequently prescribed antacid drugs were proton pump inhibitors, used by $360(8.8 \%)$ patients in cohort A and $393(7.3 \%)$ in cohort B; for antiplatelet drugs, it was acetylsalicylic acid, used by $218(5.3 \%)$ patients in cohort A and 488 (9.1\%) in cohort B.

Most cohort A $(65 \%)$ patients and the dabigatran subgroup of cohort B $(70 \%)$ were treated with dabigatran $150 \mathrm{mg}$ two times per day; the remainder were treated with dabigatran $110 \mathrm{mg}$ two times per day. In cohort A, the mean duration of previous VKA therapy was 34 months (median 19 months), with warfarin $(\mathrm{n}=2680$ $(65 \%))$ and acenocoumarol $(\mathrm{n}=1233(30 \%))$ the most frequently used VKAs.

\section{Treatment perceptions (outcomes)}

Primary outcome in patients switched from VKA to dabigatran (cohort A)

Mean PACT-Q2 treatment convenience and satisfaction scores are shown in figure $1 \mathrm{~A}$, with both scores improving significantly from baseline to V2 and V3. For treatment convenience, mean PACT-Q2 change from V1 at V2 was 20.72 (SD 21.50; $\mathrm{p}<0.001$ ), and from V1 at V3 it was 24.54 (SD 22.85; $\mathrm{p}<0.001$ ). For treatment satisfaction, mean PACT-Q2 satisfaction change from V1 at V2 was 17.60 (SD 18.76; $\mathrm{p}<0.001$ ), and from V1 at V3 it was 21.04 (SD 20.24; $\mathrm{p}<0.001)$.

Secondary outcome in patients switched from VKA to dabigatran (cohort A)

The mean changes (improvements) over time in PACT-Q2 treatment convenience and satisfaction scores between V3 and V2 were also statistically significant (figure 1A). Mean change in PACT-Q2 scores between V3 and V2 for treatment convenience was 3.62 (SD 12.98; $\mathrm{p}<0.001)$, and for treatment satisfaction it was 3.33 (SD 12.86; $<<0.001$ ).

Primary outcome in patients newly initiated on dabigatran or a VKA (cohort B)

Mean PACT-Q2 scores for treatment convenience and satisfaction were significantly higher in the dabigatran group compared with the VKA group at both V2 and V3 $(p<0.001$ for all), with treatment differences increasing over time (figure 1B). For dabigatran versus VKA, mean 
Arrhythmias and sudden death

Table 3 Physician-rated risk scores (main analysis set)

\begin{tabular}{|c|c|c|c|c|}
\hline & \multicolumn{2}{|c|}{ Cohort A (switched from VKA to dabigatran) } & \multicolumn{2}{|c|}{$\begin{array}{l}\text { Cohort B (newly initiated on dabigatran } \\
\text { or a VKA) }\end{array}$} \\
\hline & Total $(n=4100)$ & Dabigatran $(n=3179)$ & VKA $(n=2186)$ & Total $(n=5365)$ \\
\hline \multicolumn{5}{|c|}{$\mathrm{CHA}_{2} \mathrm{DS}_{2}$-VASc score (category), $\mathrm{n}(\%)$} \\
\hline High risk $(\geq 2)$ & $3619(88.3)$ & $2791(87.8)$ & $1998(91.4)$ & $4789(89.3)$ \\
\hline Low/intermediate risk $(<2)$ & $222(5.4)$ & $262(8.2)$ & $153(7.0)$ & $415(7.7)$ \\
\hline Not available & $259(6.3)$ & $126(4.0)$ & $35(1.6)$ & $161(3.0)$ \\
\hline \multicolumn{5}{|c|}{$\mathrm{CHA}_{2} \mathrm{DS}_{2}$-VASc score (result), $\mathrm{n}(\%)$} \\
\hline 0 & $13(0.3)$ & $19(0.6)$ & $14(0.6)$ & $33(0.6)$ \\
\hline 1 & $209(5.1)$ & $243(7.6)$ & $139(6.4)$ & $382(7.1)$ \\
\hline 2 & $529(12.9)$ & $599(18.8)$ & $400(18.3)$ & $999(18.6)$ \\
\hline 3 & $944(23.0)$ & $759(23.9)$ & $536(24.5)$ & $1295(24.1)$ \\
\hline 4 & $875(21.3)$ & $643(20.2)$ & $481(22.0)$ & $1124(21.0)$ \\
\hline 5 & $640(15.6)$ & $404(12.7)$ & $330(15.1)$ & $734(13.7)$ \\
\hline 6 & $362(8.8)$ & $263(8.3)$ & $150(6.9)$ & $413(7.7)$ \\
\hline 7 & $195(4.8)$ & $85(2.7)$ & 77 (3.5) & $162(3.0)$ \\
\hline 8 & $62(1.5)$ & $25(0.8)$ & $18(0.8)$ & $43(0.8)$ \\
\hline 9 & $12(0.3)$ & $13(0.4)$ & $6(0.3)$ & $19(0.4)$ \\
\hline Not available & $259(6.3)$ & $126(4.0)$ & $35(1.6)$ & $161(3.0)$ \\
\hline \multicolumn{5}{|c|}{ HAS-BLED score (category), n (\%) } \\
\hline High risk ( $\geq 3$ ) & $2429(59.2)$ & $925(29.1)$ & $685(31.3)$ & $1610(30.0)$ \\
\hline Low risk $(<3)$ & $1272(31.0)$ & $2039(64.1)$ & $1437(65.7)$ & $3476(64.8)$ \\
\hline Not available & $399(9.7)$ & $215(6.8)$ & $64(2.9)$ & $279(5.2)$ \\
\hline \multicolumn{5}{|l|}{ HAS-BLED score (result), $n$ (\%) } \\
\hline 0 & $52(1.3)$ & $166(5.2)$ & $112(5.1)$ & $278(5.2)$ \\
\hline 1 & $386(9.4)$ & $785(24.7)$ & $553(25.3)$ & $1338(24.9)$ \\
\hline 2 & $834(20.3)$ & $1088(34.2)$ & $772(35.3)$ & $1860(34.7)$ \\
\hline 3 & $1149(28.0)$ & $634(19.9)$ & $492(22.5)$ & $1126(21.0)$ \\
\hline 4 & $865(21.1)$ & $223(7.0)$ & $150(6.9)$ & $373(7.0)$ \\
\hline 5 & $313(7.6)$ & $61(1.9)$ & $39(1.8)$ & $100(1.9)$ \\
\hline 6 & $82(2.0)$ & $6(0.2)$ & $4(0.2)$ & $10(0.2)$ \\
\hline 7 & $18(0.4)$ & $1(0.0)$ & - & $1(0.0)$ \\
\hline 8 & $2(0.0)$ & - & - & - \\
\hline Not available & 399 (9.7) & $215(6.8)$ & $64(2.9)$ & 279 (5.2) \\
\hline
\end{tabular}

VKA, vitamin $\mathrm{K}$ antagonist.

treatment difference in PACT-Q2 scores for treatment convenience at V2 was 18.38 (SE 0.51), and at V3 it was 23.34 (SE 0.51). For treatment satisfaction at V2, it was 15.88 (SE 0.39), and at V3 it was 19.01 (SE 0.41). All differences in treatment convenience and satisfaction PACT-Q2 scores between treatment groups at both V2 and V3 were statistically significant $(\mathrm{p}<0.001)$.

Secondary outcome in patients newly initiated on dabigatran or a VKA (cohort B)

Table 4 summarises patient treatment expectations (PACT-Q1 items) at baseline in cohort B. Most patients were confident that their anticoagulant treatment would prevent blood clots (59\% reporting 'a lot' or 'extremely') and had moderate-to-high expectation of symptom relief (65\% reporting 'moderately', 'a lot' or 'extremely'). The majority of patients considered it important to have an anticoagulant treatment that was easy to take $(77 \%$ reporting 'a lot' or 'extremely').

\section{Safety outcomes}

Similar numbers of ADRs or serious ADRs were reported by patients in cohorts $\mathrm{A}$ and $\mathrm{B}$, and the dabigatran and VKA cohort B treatment groups (table 5). There were few severe ADRs: $12(0.3 \%)$ patients in cohort A and $21(0.4 \%)$ in cohort B (dabigatran: $14(0.4 \%)$; VKA: 7 $(0.3 \%))$. ADRs leading to discontinuation were reported in $43(1.1 \%)$ patients in cohort $\mathrm{A}$ and $36(0.7 \%)$ in 
A

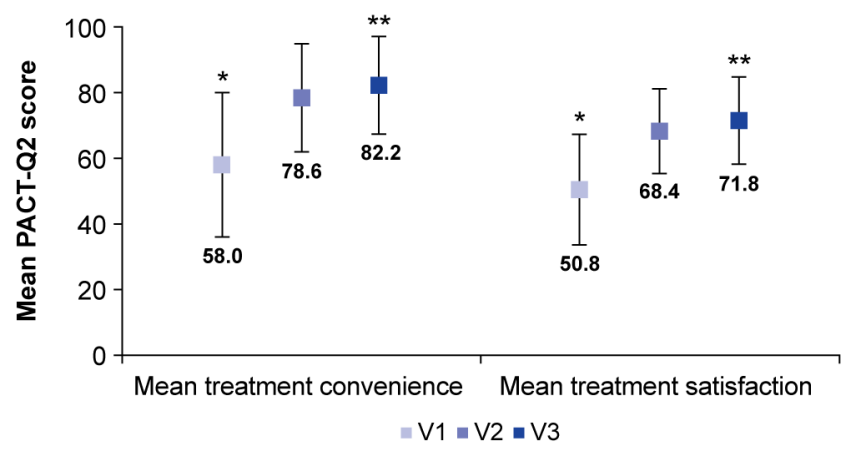

B

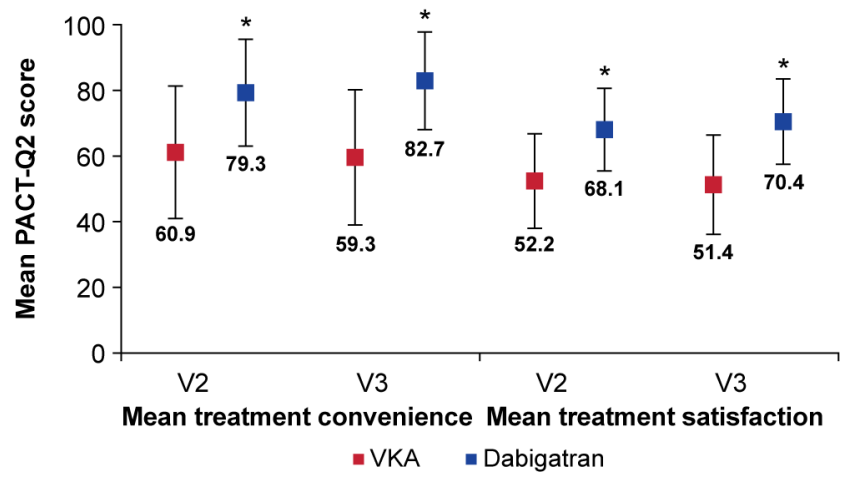

Figure 1 Mean PACT-Q2 treatment convenience and satisfaction scores. (A) Cohort $A$ (patients switched from VKA to dabigatran). ${ }^{*} \mathrm{P}<0.001 \mathrm{~V} 1$ versus $\mathrm{V} 2$ and $\mathrm{V} 1$ versus $\mathrm{V} 3$. ${ }^{* *} \mathrm{P}<0.001 \mathrm{~V} 2$ versus V3. Error bars represent SD. (B) Cohort $B$ (patients newly initiated on dabigatran or a VKA). ${ }^{*} P<0.001$ dabigatran versus VKA. Error bars represent SD. PACT-Q, Perception on Anticoagulant Treatment Questionnaire; V1, baseline; V2, initiation period; V3, continuation period; VKA, vitamin $\mathrm{K}$ antagonist.

cohort B (dabigatran: $32(1.0 \%)$; VKA: $4(0.2 \%))$. At the system organ class level, the most frequently reported ADRs leading to discontinuation were gastrointestinal (GI) disorders (the most common being abdominal pain, dyspepsia and GI haemorrhage), reported in 24 $(0.6 \%)$ patients in cohort A and $17(0.3 \%)$ in cohort B (dabigatran: $15(0.5 \%)$; VKA: $2(0.1 \%))$. Nine patients experienced ischaemic stroke: three $(0.1 \%)$ in cohort A, six $(0.1 \%)$ in cohort B (dabigatran: $5(0.2 \%)$; VKA: $1(0.0 \%))$; of these, two cohort A patients discontinued and one in the cohort B VKA group died. Few patients experienced fatal ADRs: five $(0.1 \%)$ in cohort $\mathrm{A}$ and six $(0.1 \%)$ in cohort B (dabigatran: $2(0.1 \%)$; VKA: 4 $(0.2 \%))$.

The number of bleeding ADRs was small (table 5), with the most common being GI and gingival bleeding events. Four severe bleeding ADRs (GI, tumour, haemorrhoidal and cerebral) were reported in four $(0.1 \%)$ patients in cohort B (dabigatran: $3(0.1 \%)$; VKA: 1 $(0.0 \%))$. Bleeding ADRs leading to discontinuation were reported by $13(0.3 \%)$ patients in cohort A and $20(0.4 \%)$ in cohort B (dabigatran: $18(0.6 \%)$; VKA: $2(0.1 \%))$. No patients experienced a fatal bleeding ADR.

\section{DISCUSSION}

There are limited data on patients' perceptions of longterm anticoagulation therapy in NVAF. In this observational study, which was a representative population of 9472 patients with NVAF in 11 European countries and Israel, treatment satisfaction and convenience in patients who switched from a VKA to dabigatran increased significantly from baseline over time. For those patients newly diagnosed with NVAF, treatment satisfaction and convenience were significantly higher for dabigatran compared with VKA therapy. Few, serious and severe ADRs were reported by similar numbers of patients in cohorts $A$ and $\mathrm{B}$, and in dabigatran-treated and VKA-treated cohort B patients. While numbers were low, patients receiving dabigatran versus a VKA reported more ADRs that led to treatment discontinuation, with GI events driving approximately half the discontinuations. However, no difference was observed in severe bleeding events in patients receiving dabigatran compared with VKA. Overall, the safety profile of dabigatran in the RE-SONANCE study was consistent with previous clinical dabigatran and VKA data in the AF setting. ${ }^{15-17}$

Few studies have assessed patients' perspectives of anticoagulant therapy in AF. In contrast to our findings, a substudy of the RE-LY trial observed stable healthrelated quality of life (measured using EQ-5D) scores over 12 months in all treated patients without outcome events, with comparable scores in the dabigatran and warfarin groups. ${ }^{13}$ This was unexpected, given the known complexities of warfarin treatment. In the PREFER in AF registry, within the first year of NOACs being available in Western Europe, patient-related factors influencing the switch from a VKA to a NOAC included complaints about bruising/bleeding, treatment dissatisfaction, mobility problems and anxiety/depression. ${ }^{11}$ Patients switching from a VKA to a NOAC had less hypertension, heart valve dysfunction and $\mathrm{CHA}_{2} \mathrm{DS}_{2}$-VASc scores, ${ }^{11}$ possibly reflecting caution on the part of physicians trying a new treatment option. In a small study using the validated Anti-Clot Treatment Scale, warfarin treatment was less favoured than non-warfarin treatments, including more limitations and greater feelings of burden. ${ }^{18}$

It is recognised that there is a need for improvement in educational strategies around $\mathrm{OACs}^{6}{ }^{9}$; in order to anticoagulate patients successfully with $\mathrm{AF}$ at high risk for stroke and prevent further ischaemic stroke, physicians and patients need to understand fully the rationale behind OAC treatment. Physicians must be provided with evidence regarding which treatment option best suits their patients' clinical presentation. For example, NOACs should be the first option physicians consider for preventing stroke in patients with AF (including aortic regurgitation or stenosis), although VKAs are indicated for preventing stroke in those patients with $\mathrm{AF}$ and a mechanical valve or moderate-to-severe mitral valve stenosis. ${ }^{3}$ For patients, providing educational strategies can significantly improve their OAC treatment knowledge compared with usual 
Table 4 Description of treatment expectations by the patients (PACT-Q1 items) in cohort B (patients newly initiated on dabigatran or a VKA) in the main analysis set

\begin{tabular}{|c|c|c|c|c|c|c|}
\hline PACT-Q1 item & Missing & Not at all & A little & Moderately & A lot & Extremely \\
\hline \multicolumn{7}{|c|}{ A1-How confident are you that your anticoagulant treatment will prevent blood clots? } \\
\hline Dabigatran* & 103 (3.2) & $46(1.4)$ & $230(7.2)$ & $803(25.3)$ & $1480(46.6)$ & $517(16.3)$ \\
\hline VKA† & $64(2.9)$ & $32(1.5)$ & $215(9.8)$ & $701(32.1)$ & $946(43.3)$ & $228(10.4)$ \\
\hline Overall & $167(3.1)$ & $78(1.5)$ & $445(8.3)$ & $1504(28.0)$ & 2426 (45.2) & 745 (13.9) \\
\hline \multicolumn{7}{|c|}{ A2-Do you expect that your anticoagulant treatment will relieve some of the symptoms you experience? } \\
\hline Dabigatran* & 103 (3.2) & $361(11.4)$ & $607(19.1)$ & $944(29.7)$ & $911(28.7)$ & $253(8.0)$ \\
\hline VKA† & $64(2.9)$ & 261 (11.9) & 488 (22.3) & $670(30.6)$ & $599(27.4)$ & $104(4.8)$ \\
\hline Overall & $167(3.1)$ & $622(11.6)$ & $1095(20.4)$ & $1614(30.1)$ & $1510(28.1)$ & $357(6.7)$ \\
\hline \multicolumn{7}{|c|}{ A3-Do you expect that your anticoagulant treatment will cause side effects such as minor bruises or bleeding? } \\
\hline Dabigatran* & $103(3.2)$ & $487(15.3)$ & $1201(37.8)$ & $1012(31.8)$ & $320(10.1)$ & $56(1.8)$ \\
\hline VKA† & $64(2.9)$ & $250(11.4)$ & $812(37.1)$ & $772(35.3)$ & $253(11.6)$ & $35(1.6)$ \\
\hline Overall & $167(3.1)$ & $737(13.7)$ & $2013(37.5)$ & $1784(33.3)$ & $573(10.7)$ & $91(1.7)$ \\
\hline \multicolumn{7}{|c|}{ A4-How important is it for you to have an anticoagulant treatment that is easy to take? } \\
\hline Dabigatran* & $103(3.2)$ & $63(2.0)$ & $106(3.3)$ & $333(10.5)$ & $1676(52.7)$ & $898(28.2)$ \\
\hline VKA† & $64(2.9)$ & $32(1.5)$ & $123(5.6)$ & $417(19.1)$ & $1153(52.7)$ & $397(18.2)$ \\
\hline Overall & $167(3.1)$ & $95(1.8)$ & $229(4.3)$ & $750(14.0)$ & $2829(52.7)$ & $1295(24.1)$ \\
\hline \multicolumn{7}{|c|}{ A5—How concerned are you about making mistakes when taking your anticoagulant treatment? } \\
\hline Dabigatran* & $103(3.2)$ & $575(18.1)$ & $698(22.0)$ & $767(24.1)$ & $804(25.3)$ & $232(7.3)$ \\
\hline VKA† & $64(2.9)$ & $208(9.5)$ & $491(22.5)$ & $646(29.6)$ & $607(27.8)$ & $170(7.8)$ \\
\hline Overall & $167(3.1)$ & $783(14.6)$ & $1189(22.2)$ & $1413(26.3)$ & $1411(26.3)$ & $402(7.5)$ \\
\hline \multicolumn{7}{|c|}{ A6- How important is it for you to take care of your anticoagulant treatment by yourself? } \\
\hline Dabigatran* & $103(3.2)$ & $68(2.1)$ & $157(4.9)$ & $398(12.5)$ & $1599(50.3)$ & $854(26.9)$ \\
\hline VKA† & $64(2.9)$ & $48(2.2)$ & $139(6.4)$ & $456(20.9)$ & $1097(50.2)$ & $382(17.5)$ \\
\hline Overall & $167(3.1)$ & $116(2.2)$ & $296(5.5)$ & $854(15.9)$ & $2696(50.3)$ & $1236(23.0)$ \\
\hline \multicolumn{7}{|c|}{ A7- How concerned are you about how much you may have to pay for your anticoagulant treatment? } \\
\hline Dabigatran* & $103(3.2)$ & $398(12.5)$ & $495(15.6)$ & $1057(33.2)$ & $780(24.5)$ & $346(10.9)$ \\
\hline VKA† & $64(2.9)$ & $183(8.4)$ & $186(8.5)$ & $396(18.1)$ & $824(37.7)$ & $533(24.4)$ \\
\hline Overall & $167(3.1)$ & $581(10.8)$ & $681(12.7)$ & $1453(27.1)$ & $1604(29.9)$ & $879(16.4)$ \\
\hline
\end{tabular}

All data are $\mathrm{n}(\%)$.

*Dabigatran: $n=3179$.

†VKA: $\mathrm{n}=2186$.

$\ddagger$ Total: $n=5365$.

PACT-Q, Perception on Anticoagulant Treatment Questionnaire; VKA, vitamin K antagonist.

care. ${ }^{8}$ By regularly evaluating knowledge gaps (eg, international normalised ratio target ranges, which concomitant medications should be avoided and recognising medical complications such as stroke or bleeding), the most appropriate educational programmes can be provided. Such strategies have been shown to increase patients' use of $\mathrm{OACs}$ significantly ${ }^{5}$ and the number of patients achieving time within therapeutic range. ${ }^{7}$ Furthermore, educational strategies can significantly reduce the risk of recurrent stroke compared with usual care. ${ }^{5}$

Other studies have observed higher treatment satisfaction among NOAC versus warfarin users. ${ }^{19-22}$ It is reasonable to extrapolate that improving anticoagulation treatment satisfaction may increase patients' adherence to treatment and improve outcomes. Open discussions with patients can help to identify potential barriers to therapy; if patients are involved in their treatment decisions, they may be more likely to take responsibility for their treatment, thereby improving adherence. ${ }^{23}$ However, despite improved treatment satisfaction with NOACs versus VKAs, recent observational data noted comparable adherence. ${ }^{21}{ }^{22}$ The paucity of data regarding treatment satisfaction and outcomes means that further studies are needed to assess the impact of improved patient perceptions.

A strength of this study is that it used the PACT-Q, which is a validated and specific treatment satisfaction instrument for thromboembolic patients with anticoagulant treatment, and is available in numerous languages. ${ }^{10} 14$ While there are inherent limitations associated with any observational study, the large patient population recruited in 


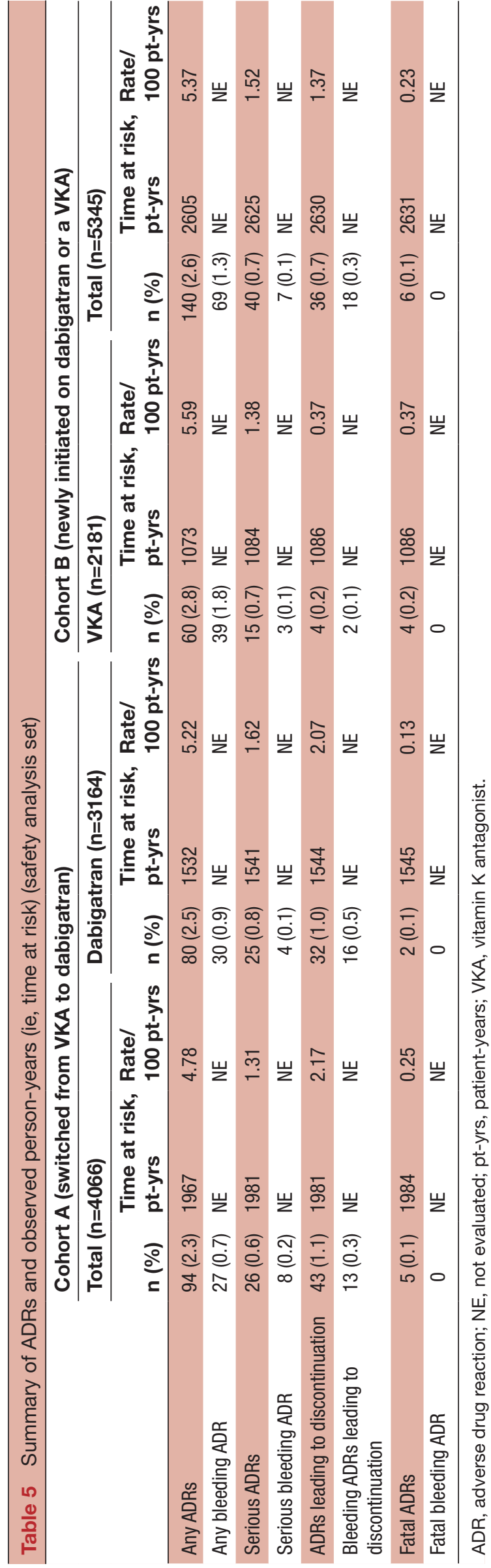

this non-interventional study is representative of patients receiving an OAC for stroke prevention in NVAF. Study limitations include the role of reimbursement, which could affect overall treatment adherence and satisfaction, and is highly dependent on a patient's financial and socioeconomic status. Patient selection bias may also be the reason why only one-third of patients were over 75 years; elderly patients, who could benefit from switching from VKA to dabigatran, may not have been included due to the perception that they might not be able to understand the questionnaires fully. Treatment bias may also have been introduced, as patients might subconsciously consider a new therapy as better. Additionally, due to the real-world nature of the study, patient follow-up was based on routine care instead of a stringent visit schedule, as used in clinical trials. Therefore, there was considerable variation between patient visits from baseline during the study follow-up. To better accommodate this and ensure that most of the collected data could be included in the analysis, more relaxed time windows were defined for V2 and V3. The balance between time window thresholds and potential misclassification was assessed, and it was decided to apply 'consecutive' thresholds between V2 and V3 to capture as much of the data as possible, and since the number of patients with extreme visit times was low. Finally, patients may not always be willing to write their honest opinions on a questionnaire or tell the doctor what they really think about the therapy. Although not aimed at assessing patient outcome, the safety profile of the OACs were comparable.

\section{CONCLUSIONS}

Switching to dabigatran from long-term VKA therapy or newly initiated dabigatran is associated with improved patient treatment convenience and satisfaction compared with VKA therapy. This may be important in the prevention of stroke and systemic embolism in patients with $\mathrm{AF}$ at moderate-to-high risk.

\section{Author affiliations}

${ }^{1}$ Cardiology, University and Emergency Hospital of Bucharest, Bucharest, Other, Romania

${ }^{2}$ Department of Internal Medicine, I.M. Sechenov First Moscow State Medical University (Sechenov University), Moscow, Russian Federation

${ }^{3}$ Department of Cardiology, University Clinic of Internal Medicine II, Medical University of Vienna, Vienna, Austria

${ }^{4} 1$ st Department of Internal Medicine (Cardiology/Nephrology), Tolna County

'Balassa Janos' Teaching Hospital, Szekszard, Hungary

${ }^{5}$ Boehringer Ingelheim RCV GmbH \& Co. KG, Vienna, Austria

${ }^{6}$ Department of Cardiology, National Cardiology Hospital, Sofia, Bulgaria

${ }^{7}$ Department of Cardiology, North Estonia Medical Centre Foundation, Tallinn,

Estonia

${ }^{8}$ Department of Cardiology, Clinical Centre of Serbia, Belgrade, Serbia

${ }^{9}$ Cardiology Department, Riga Stradins University, Riga, Latvia

${ }^{10}$ Biostatistics and Data Sciences, Boehringer Ingelheim Pharmaceuticals, Inc, Ridgefield, Connecticut, USA

${ }^{11}$ First Department of Cardiology, Silesian Medical University, Katowice, Poland

${ }^{12}$ Faculty of Medicine in Hradec Kralové, Charles University, Broumov, Czech

Republic

${ }^{13}$ Clalit Health Services, Bonen Clinic, Haifa, Israel

${ }^{14}$ Department of Cardiology, General Hospital Celje, Celje, Slovenia 
Acknowledgements The authors would like to thank all the patients who participated in this study. Editorial assistance consisting of formatting and collation of author comments was provided by PAREXEL, with funding from Boehringer Ingelheim.

Contributors AS is accountable for work in data collection. MT-G led the coordination of the study at her institution. All authors contributed to the drafting, interpretation and reviewing of the manuscript content and provided final approval of the manuscript.

Funding This study was funded by Boehringer Ingelheim International GmbH. Competing interests DV reports grants and personal fees from Boehringer Ingelheim, during the conduct of the study; grants and personal fees from Bayer, Bristol-Myers Squibb, Daiichi Sankyo, Pfizer and J\&J outside the submitted work. DN reports research and speaker fees for Boehringer Ingelheim and speaker fees for Bayer, Pfizer and Takeda. JB-K, NG, DS, AS, TV and MV report no conflicts of interest. BB has received speaker/consultancy fees from Bayer, Boehringer Ingelheim, Berlin-Chemie/Menarini, KRKA, Novartis, Pfizer, Sandoz and Sanofi. MC, AM and WT are employees of Boehringer Ingelheim. PP has received educational grants from Boehringer Ingelheim. MT-G reports personal fees from Boehringer Ingelheim during the conduct of this study; personal fees and non-financial support from Boehringer Ingelheim outside the submitted work and personal fees from Bayer. JV reports personal fees and non-financial support from Bayer, Boehringer Ingelheim, MSD, Pfizer and PRO.MED.CZ outside the submitted work.

Patient consent for publication Not required.

Ethics approval This study was carried out in accordance with the Declaration of Helsinki, International Conference of Harmonisation Tripartite Guideline, Good Clinical Practice, Guidelines for Good Epidemiological Practice and Good Pharmacoepidemiology Practice. The study was initiated in centres once approved by the respective Institutional Review Board/Independent Ethics Committee and competent authority, according to national and international regulations.

Provenance and peer review Not commissioned; externally peer reviewed. Data availability statement Data are available upon reasonable request. To ensure independent interpretation of clinical study results, Boehringer Ingelheim grants all external authors access to all relevant material, including participantlevel clinical study data, and relevant material as needed by them to fulfil their role and obligations as authors under the ICMJE criteria. Furthermore, clinical study documents (eg, study report, study protocol, statistical analysis plan) and participant clinical study data are available to be shared after publication of the primary manuscript in a peer-reviewed journal and if regulatory activities are complete and other criteria met per the Boehringer Ingelheim Policy on Transparency and Publication of Clinical Study Data: https://trials.boehringeringelheim.com/transparency_policy.html. Prior to providing access, documents will be examined and, if necessary, redacted and the data will be de-identified to protect the personal data of study participants and personnel and to respect the boundaries of the informed consent of the study participants. Clinical Study Reports and Related Clinical Documents can be requested via this link: https:// trials.boehringer-ingelheim.com/trial_results/clinical_submission_documents.html. All such requests will be governed by a Document Sharing Agreement. Bona fide qualified scientific and medical researchers may request access to de-identified, analysable participant clinical study data with corresponding documentation describing the structure and content of the data sets. On approval, and governed by a Data Sharing Agreement, data are shared in a secured data-access system for a limited period of 1 year, which may be extended upon request. Researchers should use https://clinicalstudydatarequest.com to request access to study data.

Open access This is an open access article distributed in accordance with the Creative Commons Attribution 4.0 Unported (CC BY 4.0) license, which permits others to copy, redistribute, remix, transform and build upon this work for any purpose, provided the original work is properly cited, a link to the licence is given, and indication of whether changes were made. See: https://creativecommons.org/ licenses/by/4.0/.

\section{ORCID iD}

Alexey Medvedchikov http://orcid.org/0000-0001-8134-454X

\section{REFERENCES}

1 Ogilvie IM, Newton N, Welner SA, et al. Underuse of oral anticoagulants in atrial fibrillation: a systematic review. Am J Med 2010;123:638-45.

2 Camm AJ, Lip GYH, De Caterina R, et al. 2012 focused update of the ESC Guidelines for the management of atrial fibrillation: an update of the 2010 ESC Guidelines for the management of atrial fibrillation--developed with the special contribution of the European Heart Rhythm Association. Europace 2012;14:1385-413.

3 Kirchhof P, Benussi S, Kotecha D, et al. 2016 ESC guidelines for the management of atrial fibrillation developed in collaboration with EACTS. Europace 2016;18:1609-78.

4 Steffel J, Verhamme P, Potpara TS, et al. The 2018 European Heart Rhythm Association Practical Guide on the use of non-vitamin K antagonist oral anticoagulants in patients with atrial fibrillation. Eur Heart J 2018:39:1330-93.

5 Vinereanu D, Lopes RD, Bahit MC, et al. A multifaceted intervention to improve treatment with oral anticoagulants in atrial fibrillation (IMPACT-AF): an international, cluster-randomised trial. Lancet 2017;390:1737-46.

6 Amara W, Larsen TB, Sciaraffia E, et al. Patients' attitude and knowledge about oral anticoagulation therapy: results of a selfassessment survey in patients with atrial fibrillation conducted by the European Heart Rhythm Association. Europace 2016;18:151-5.

7 Clarkesmith DE, Pattison HM, Lip GYH, et al. Educational intervention improves anticoagulation control in atrial fibrillation patients: the TREAT randomised trial. PLoS One 2013;8:e74037.

8 Maikranz V, Siebenhofer A, Ulrich L-R, et al. Does a complex intervention increase patient knowledge about oral anticoagulation? - a cluster-randomised controlled trial. BMC Fam Pract 2017;18:15.

9 Glauser TA, Barnes J, Nevins H, et al. The educational needs of clinicians regarding anticoagulation therapy for prevention of thromboembolism and stroke in patients with atrial fibrillation. $A m \mathrm{~J}$ Med Qual 2016;31:38-46.

10 Prins MH, Marrel A, Carita P, et al. Multinational development of a questionnaire assessing patient satisfaction with anticoagulant treatment: the 'Perception of Anticoagulant Treatment Questionnaire (PACT-Q). Health Qual Life Outcomes 2009;7:9.

11 De Caterina $\mathrm{R}$, Brüggenjürgen $\mathrm{B}$, Darius $\mathrm{H}$, et al. Quality of life and patient satisfaction in patients with atrial fibrillation on stable vitamin $\mathrm{K}$ antagonist treatment or switched to a non-vitamin $\mathrm{K}$ antagonist oral anticoagulant during a 1-year follow-up: a PREFER in AF Registry substudy. Arch Cardiovasc Dis 2018;111:74-84.

12 Lane DA, Meyerhoff J, Rohner U, et al. Atrial fibrillation patient preferences for oral anticoagulation and stroke knowledge: results of a conjoint analysis. Clin Cardiol 2018;41:855-61.

13 Monz BU, Connolly SJ, Korhonen M, et al. Assessing the impact of dabigatran and warfarin on health-related quality of life: results from an RE-LY sub-study. Int J Cardiol 2013;168:2540-7.

14 Prins $\mathrm{MH}$, Guillemin I, Gilet $\mathrm{H}$, et al. Scoring and psychometric validation of the Perception of Anticoagulant Treatment Questionnaire (PACT-Q). Health Qual Life Outcomes 2009;7:30.

15 Connolly SJ, Ezekowitz MD, Yusuf S, et al. Dabigatran versus warfarin in patients with atrial fibrillation. $N$ Engl $\mathrm{J}$ Med 2009;361:1139-51.

16 Calkins H, Willems S, Gerstenfeld EP, et al. Uninterrupted dabigatran versus warfarin for ablation in atrial fibrillation. $N$ Engl $J$ Med 2017;376:1627-36.

17 Cannon CP, Lip GYH, Oldgren J. Dual antithrombotic therapy with dabigatran after $\mathrm{PCl}$ in atrial fibrillation. N Engl J Med 2018;378:485-6.

18 Coleman $\mathrm{Cl}$, Coleman SM, Vanderpoel J, et al. Patient satisfaction with warfarin- and non-warfarin-containing thromboprophylaxis regimens for atrial fibrillation. $J$ Investig Med 2013;61:878-81.

19 Okumura Y, Yokoyama K, Matsumoto N, et al. Patient satisfaction with direct oral anticoagulants and warfarin. Int Heart $J$ 2018;59:1266-74.

20 Prins MH, Bamber L, Cano SJ, et al. Patient-reported treatment satisfaction with oral rivaroxaban versus standard therapy in the treatment of pulmonary embolism; results from the EINSTEIN PE trial. Thromb Res 2015;135:281-8.

21 Benzimra M, Bonnamour B, Duracinsky M, et al. Real-life experience of quality of life, treatment satisfaction, and adherence in patients receiving oral anticoagulants for atrial fibrillation. Patient Prefer Adherence 2018;12:79-87.

22 Keita I, Aubin-Auger I, Lalanne C, et al. Assessment of quality of life, satisfaction with anticoagulation therapy, and adherence to treatment in patients receiving long-course vitamin $\mathrm{K}$ antagonists or direct oral anticoagulants for venous thromboembolism. Patient Prefer Adherence 2017:11:1625-34.

23 Williams B, Mancia G, Spiering W, et al. 2018 ESC/ESH guidelines for the management of arterial hypertension: the task force for the management of arterial hypertension of the European Society of Cardiology and the European Society of Hypertension: the task force for the management of arterial hypertension of the European Society of Cardiology and the European Society of Hypertension. $J$ Hypertens 2018;36:1953-2041. 